\title{
String Searching in Referentially Compressed Genomes
}

\author{
Sebastian Wandelt ${ }^{1}$ and Ulf Leser ${ }^{1}$ \\ ${ }^{1}$ Humboldt-Universität zu Berlin, Wissensmanagement in der Bioinformatik, \\ Rudower Chaussee 25, 12489 Berlin, Germany \\ \{wandelt, leser\}@informatik.hu-berlin.de
}

Keywords: Genome compression, referential compression, string search

Abstract: Background:Improved sequencing techniques have led to large amounts of biological sequence data. One of the challenges in managing sequence data is efficient storage. Recently, referential compression schemes, storing only the differences between a to-be-compressed input and a known reference sequence, gained a lot of interest in this field. However, so far sequences always have to be decompressed prior to an analysis. There is a need for algorithms working on compressed data directly, avoiding costly decompression.

Summary:In our work, we address this problem by proposing an algorithm for exact string search over compressed data. The algorithm works directly on referentially compressed genome sequences, without needing an index for each genome and only using partial decompression.

Results:Our string search algorithm for referentially compressed genomes performs exact string matching for large sets of genomes faster than using an index structure, e.g. suffix trees, for each genome, especially for short queries. We think that this is an important step towards space and runtime efficient management of large biological data sets.

\section{Introduction}

The development of novel high-throughput DNA sequencing techniques has led to an ever increasing flood of data. Current techniques, usually summarized under the term second generation sequencing (SGS), are able to produce roughly the same amount of data in about a week at a current cost of roughly 2000 USD. Is is predicted, that third generation sequencing deliver a further speed-up, reducing the time and price for sequencing a human genome from weeks to days and from thousands to under a hundred USD, respectively (Schadt et al., 2010). As a concrete example, the UK-based Wellcome Trust Sequencing Center recently reported that its throughput in terms of DNA sequences has risen from 100KB/ day in the times of the human genome project to currently 1TB/day (Chiang et al., 2011).

A human genome consists of 23 chromosomes. Each chromosome is a sequence of 50.000 .000 and 250.000.000 nucleotides: A(denin), C(ytosin), $\mathrm{G}$ (uanin), or T(hymin). In order to store a complete genome of a human being, one needs more than 3 GB (uncompressed). Sequence compression is one key technology to cope with the increasing flood of DNA sequences (Pennisi, 2011).

Substitutional or statistic compression schemes can reduce the space requirements by up to $6: 1$ (one base is encoded with up to $1.3 \mathrm{Bit}$ ) (Antoniou et al., 2010; Pratas and Pinho, 2011). However, in many projects only genomes from one species are considered. This means that projects often deal with hundreds of highly similar genomes; for instance, two randomly selected human genomes are identical to an estimated $99.9 \%$. This observation is exploited by so-called referential compression schemes, which only encode the differences of an input sequence with respect to a pre-selected reference sequence. Using space-efficient encoding of differences and clever algorithms for finding long stretches of DNA without differences, the best current referential compression algorithm we are aware of reports a compression rates of up to 500:1 (Deorowicz and Grabowski, 2011), yielding 4-8 MB per genome.

In the future, many research facilities will need to manage and analyze large sets of genomes. We think that these genomes have to be referentially compressed, in order to overcome the I/O- and storage bottlenecks of todays computing systems in bioinformatics(Kahn, 2011).

One important type of analysis is string search, i.e. finding matching parts of the sequence with respect to a given query. In this paper, we discuss string searching on large sets of genomes $G$ : Given an input query 
Table 1: Rough storage requirements for approximate string search over 1000 genomes

\begin{tabular}{cccc}
\hline Element & index-based & index-less & Referential search \\
\hline Compressed genomes & $6 \mathrm{~GB}$ & $6 \mathrm{~GB}$ & $6 \mathrm{~GB}$ \\
Index compressed genomes & $3+\mathrm{TB}$ & $0 \mathrm{~GB}$ & $0 \mathrm{~GB}$ \\
Uncompressed reference sequence & $0 \mathrm{~GB}$ & $0 \mathrm{~GB}$ & $3 \mathrm{~GB}$ \\
Index for reference sequence & $0 \mathrm{~GB}$ & $0 \mathrm{~GB}$ & $7 \mathrm{~GB}$ \\
\hline \hline Sum & $3+\mathrm{TB}$ & $6 \mathrm{~GB}$ & $16 \mathrm{~GB}$
\end{tabular}

string $q$, the task is to find all matches of $q$ in each genome in $G$.

The traditional approach is to perform the search on each genome in $G$ using an index. However, since these indices are often bigger than the uncompressed input, this naive approach seems to be not feasible. For instance, the raw data of 1000 uncompressed genomes already needs 3 TB. Having an index structure, e.g. a compressed suffix tree, for each genome will increase the data size further by factors of 3-5 or more(Vlimki et al., 2009). Therefore, we think that using one index structures for each referentially compressed genome is not feasible in large-scale projects.

Another approach is to decompress all strings in $G$, and perform index-less string search.

In this paper, we propose a third solution: searching within compressed sequences. All input sequences were referentially compressed with respect to a reference sequence, and for the compression one usually needs an index structure for that reference. Our idea is to use the existing index structure of the reference for a string search algorithm on all compressed genomes in $G$. Since all genomes in $G$ were referentially compressed with respect to the reference, large substrings of the reference will occur in the genomes in $G$ as well, interrupted by SNPs and longer variations. Therefore, our referential search algorithm solves the string matching problem as follows: 1) It finds all exact matches in the reference sequence using an index of the reference only and 2) it uses these matches for identifying all exact matches in genomes in $G$.

The space requirements for all three approaches are compared in Table 1. We report on the run times of different approaches in detail below.

The remaining paper is structured as follows. In Section 2, we discuss related work on compression of genome sequences. We introduce a general referential compression algorithm in Section 3 and propose an exact string search algorithm for referentially compressed sequences in Section 4. In Section ??, we extend the exact string search algorithm for approximate string searching. A preliminary evaluation is given in Section 5. The paper concludes in Section 6.

The remaining part of the paper is structured as follows. In Section 2, we discuss related work on compression of genome sequences. We introduce a general referential compression algorithm in Section 3 and propose a string search algorithm of referentially compressed sequences in Section 4. A preliminary evaluation is given in Section 5 . The paper is concluded with Section 6.

\section{Related Work}

The increasing number of (re-)sequenced genomes has lead to many compression algorithms. We only review losless compression schemes here. In general, these compression algorithms can be separated into bit-manipulating, dictionary-based, statistical, and referential approaches:

- Bit manipulation algorithms exploit encodings of two or more symbols into one byte (Vey, 2009; Bharti et al., 2011; Mishra et al., 2010).

- Dictionary-based or substitutional algorithms replace long repeated substrings by references to a dictionary built at runtime (Kuruppu et al., 2012; Antoniou et al., 2010; Kaipa et al., 2010).

- Statistical or entropy encoding algorithms derive a probabilistic model from the input. Based on partial matches of subsets of the input, this model predicts the next symbols in the sequence. High compression rates are possible if the model always indicates high probabilities for the next symbol, i.e. if the prediction is reliable(Duc Cao et al., 2007; Pratas and Pinho, 2011).

- Referential or reference-based approaches are similar to dictionary-based techniques, as they replace long substrings with references. However, these references point to external sequences, which are not part of the to-be-compressed input data (Brandon et al., 2009; Kuruppu et al., 2010; Pande and Matani, 2011; Grabowski and Deorowicz, 2011).

There is additional work on read compression, e.g. (Bhola et al., 2011) and (Wan et al., 2011). The main problem in read compression is the compression of 


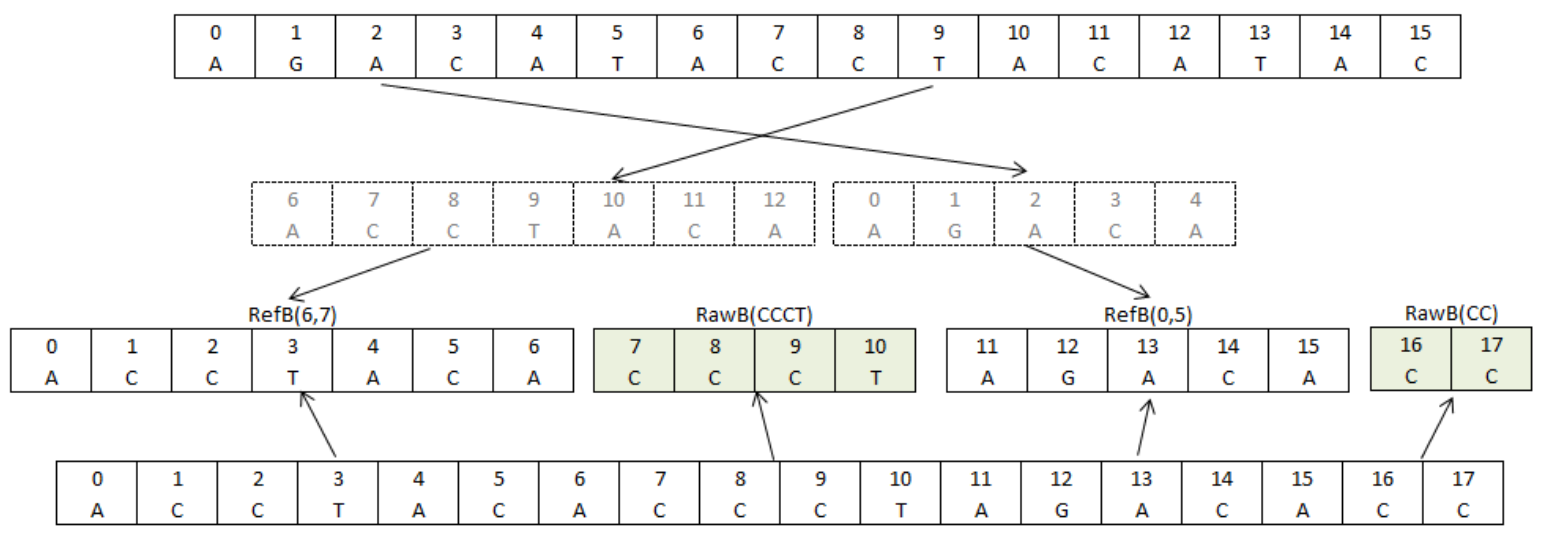

Figure 1: Example for relative compression

quality scores, e.g. (Chen et al., 2011). However, for the compression of whole genome sequences, quality scores are, so far, not important. This might change in the near future.

There has been a lot of research focused on efficient string search in collections of string. Standard techniques are Boyer-Moore(Boyer and Moore, 1977) and the use of suffix trees(Ukkonen, 1995). There exists also work on managing and searching genomic sequences in large databases, e.g. (Altschul et al., 1990; Kent, 2002; Hunt et al., 2002). However, to the best of our knowledge there exists no work on string search over referentially compressed genomic sequences.

\section{Referential Compression and Decompression}

We denote strings with $s, t$. The length of a string $s$ is denoted with $|s|$ and the substring starting at position $i$ with length $n$ is denoted $s(i, n) . s(i)$ is an abbreviation for $s(i, 1)$. All positions in a string are zero-based, i.e. the first character is accessed by $s(0)$. The concatenation of two strings $s$ and $t$ is denoted with $s \circ t$. Although a genome can be encoded with four characters, i.e. A,C,G, and T, we allow arbitrary symbols. For instance, symbol $N$ is often used to indicate an unknown base. Given two strings $s$ and $t$, the longest prefix-suffix match of $s$ in $t$, is the longest string $t_{m}$, such that $t=t_{1} \circ t_{m} \circ t_{2}$ and $s\left(0,\left|t_{m}\right|\right)=t_{m}$.

In referential compression, one or more data sequences are compressed with respect to a reference sequence, by only encoding differences between the input and the reference. This yields loss-less compression, i.e. based on the reference sequence and the difference description it is possible to recover the data sequences. A referential compression algorithm needs to generate a set of referential matches with respect to the reference. The output of our referential compression algorithm is a file of compression blocks, such that each block is one of the following:

- Referential block $\operatorname{RefB}(i, j)$ : The data sequence matches the reference sequence at position $i$ for $j$ characters

- Raw block $\operatorname{RawB}(s)$ : A string $s$ is encoded raw (for instance if there is no good matching reference block).

Consider the reference sequence:

$$
\text { reference }=\text { AGACATACCTACATAC }
$$

together with the data sequence

$$
\text { input }=\text { ACCTACACCCTAGACACC, }
$$

as shown in Figure 1. One compression of input with respect to reference is

$$
\begin{aligned}
\text { comp }= & (\operatorname{RefB}(6,7), \operatorname{RawB}(C C C T), \operatorname{RefB}(0,5), \\
& \operatorname{RawB}(C C)) .
\end{aligned}
$$

The first seven symbols of input match the symbols 6 to 12 and are encoded as a referential block $\operatorname{RefB}(6,7)$, followed by a raw block, $\operatorname{RawB}(C C C T)$. The raw block is followed by another referential block, $\operatorname{Ref} B(0,5)$, indicating that the input matches the first six symbols of the reference sequence. The last compression block is a raw block, $\operatorname{RawB}(C C)$. Altogether, the input sequence is encoded with five compression blocks.

The compression ratio is dominated by the syntactical representation of referential blocks, especially the efficient encoding of integer values, and the number and length of referential matches. In the remaining part of the paper, we do not discuss efficient encodings of compression schemes further, since there exists plenty of research on that topic already, for instance (Brandon et al., 2009) and (Daily et al., 2010). 
To efficiently find referential blocks, we use suffix trees for the reference sequence. The suffix trees of a reference sequence allows us to find longest prefixsuffix matches of parts of the data sequences with respect to the the reference genome.

In the following, we present our compression algorithm for genome sequences in detail. Algorithms do not show range checks for the sake of readability. Algorithm 1 assumes one data sequence in Input (as a string of symbols). The input string is traversed from left to right, and depending on the current symbols in the input and in the reference block, different subroutines are executed. The function FIND-MATCH is used to find the longest prefix-suffix match of the current input position with respect to the reference. If the match is shorter than $M I N$ symbols, or starts with a non-base symbol, the function ENCODE-RAW is used for raw encoding of the next input symbols. Otherwise, the function ENCODE-REF is used for referential encoding of the next input symbols.

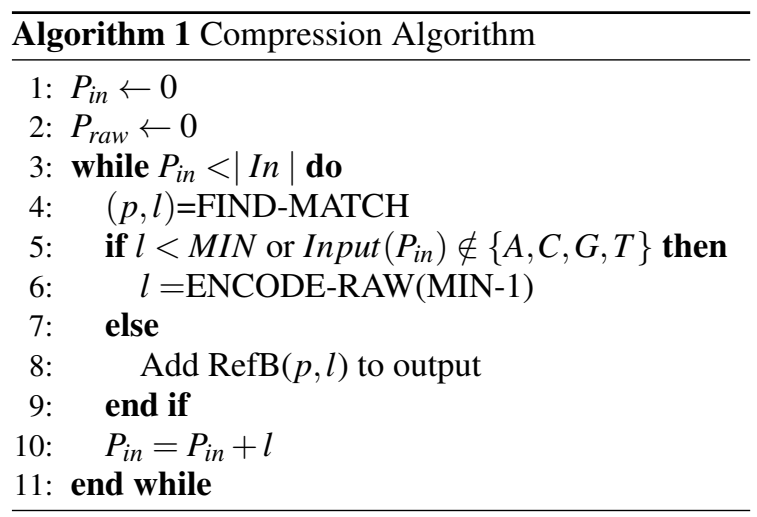

Matches are required to have at least $M I N$ characters, in order to avoid spurious matches. Our experiments have shown that the mere length of genomes, e.g. human genomes, causes a lot of unrelated matches with less than 20-25 characters. Furthermore, the compression gain is very small for such short matches, while finding them is expensive (each of them needs one index-lookup). The encoding of a raw sequence in Algorithm 2 is straight-forward: the string Raws is filled with symbols from the input until a normal base is found or a length-constraint is violated. In the end, $\operatorname{Raw}(R a w s)$ is added to the output.

We show the compression process for the compression of (see Figure 1)

$$
\text { In = ACCTACACCCTAGACACC }
$$

with respect to the reference sequence

$$
\operatorname{Ref}=\text { AGACATACCTACATAC. }
$$

Lets assume that $M I N=5$. First, the longest match for the start of In is looked up in Ref and

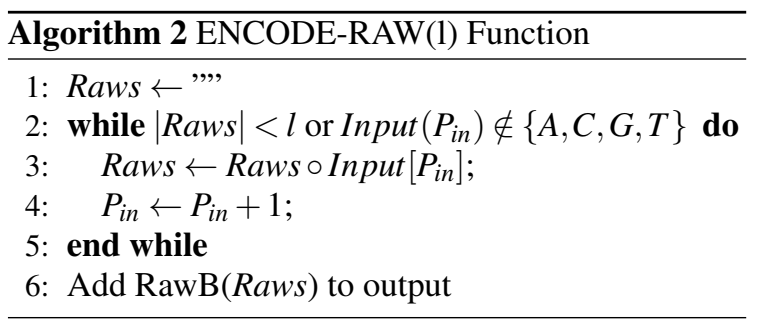

$(6,7)$ is returned (for matching ACCTACA in the reference). $\operatorname{Ref} B(6,7)$ is added to the output. Afterwards the input position $P_{\text {in }}$ is 7, and the algorithm tries to find a new match for CCCTA.... The longest local match is $(7,2)$, which is shorter than five, and therefore $\operatorname{RawB}(C C C T)$ is added to the output. Afterwards the input position $P_{i n}$ is 11 , and the algorithm tries to find a new match for $A G A C \ldots$... The result of FIND-MATCH is $(0,5)$, such that $\operatorname{Re} f B(0,5)$ is added to the output. Next, the remaining string $C C$ is added as a raw block $\operatorname{RawB}(C C)$ to the output.

Decompression of the referentially encoded input sequences is straightforward. Basically, all compression blocks are unfolded according to their definition (raw or referential block). For decompression of data sequences we do not need the compressed suffix trees any longer, but only the reference sequence.

\section{String search algorithm}

There exists plenty of work on string search algorithms for (not compressed) strings. Usually, an index structure is computed, for instance, a suffix tree. These index structures allow for fast access to substrings.

However, in our case, it is not feasible to have index structures over all compressed genome sequences. For the sake of efficiency and for minimizing storage requirements, we want to avoid computing these index structures (which are usually orders of magnitudes bigger than the data sequences, see Table 1). Thus, we have an index structure for the reference sequence only. In the following, we show how the index structure for the reference sequence can be used to efficiently find matches over referentially (w.r.t. that reference) compressed sequences.

Assume that we want to search for the query $q=$ $A C C$ in the input Input from the example above. The situation is depicted in Figure 2. Matches for the query $A C C$ can be inside a compression block or be overlapping more than one block. More formally, if a string $s$ is a subset of another (referentially compressed) string $t, s$ must either: 


\begin{tabular}{|c|c|c|c|c|c|c|c|c|c|c|c|c|c|c|c|}
\hline 0 & 1 & 2 & 3 & 4 & 5 & 6 & 7 & 8 & 9 & 10 & 11 & 12 & 13 & 14 & 15 \\
$\mathrm{~A}$ & $\mathrm{G}$ & $\mathrm{A}$ & $\mathrm{C}$ & $\mathrm{A}$ & $\mathrm{T}$ & $\mathrm{A}$ & $\mathrm{C}$ & $\mathrm{C}$ & $\mathrm{T}$ & $\mathrm{A}$ & $\mathrm{C}$ & $\mathrm{A}$ & $\mathrm{T}$ & $\mathrm{A}$ & $\mathrm{C}$ \\
\hline
\end{tabular}

Compressed Input:
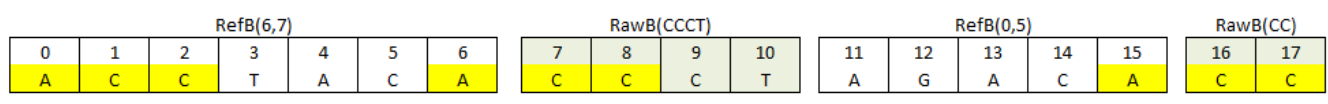

Figure 2: Example for search of $A C C$

1. be a substring of one referential block (actually the dereferenced referential block),

2. be a substring of one raw block, or

3. be an string overlapping two or more compression blocks.

Since this list contains all possible cases, we can find all matches for a query $q$ by solving each sub problem. The first kind of substrings is easy to find, since we have an index on the reference sequence. The second kind of substrings can be found by searching the raw blocks. The overlapping substrings can be found by thoughtful investigation and partial decompression of subsequences next to the beginning and end of each block. All three steps are explained below. The list of compression entries for our example is

$$
\begin{aligned}
\text { Entries }= & (\operatorname{RefB}(6,7), \operatorname{RawB}(C C C T), \operatorname{RefB}(0,5), \\
& \operatorname{RawB}(C C)) .
\end{aligned}
$$

The set of relative match entries is

$$
\text { RelMatchEntries }=\{\operatorname{RefB}(6,7), \operatorname{RefB}(0,5)\} .
$$

The set of raw entries is

$$
\text { RawEntries }=\{\operatorname{RawB}(C C C T), \operatorname{RawB}(C C)\} .
$$

\subsection{Finding Matches inside Referential Blocks}

Given the matches for $q$ from the reference sequence, we can conclude matches inside relative match entries immediately. Let RefMatches = $\{(i, j) \mid \operatorname{Reference}(i, j)=q\}$, which can be easily computed by using the suffix tree for Reference. In order to find all positions of $q$ occurring inside referential blocks, we need to find all intervals in RefMatches which are included in at least one interval of RelMatchEntries. In our example, the result would be the set $\{\operatorname{Ref} B(6,7)\}$, since we have Reference $(6,3)=A C C$ and the string interval $(6,7)$ includes the string interval $(6,2)$.

Intervals could be naively computed by pairwise comparison of each block in RelMatchEntries with each block in RefMatches. However, this is expenisve, especially if we have many data sequences and therefore many sets of RMEntries which need to be checked against one RefMatches set. A better approach is to order the reference matches in RefMatches by position of the match, e.g. this yields $((0,5),(6,7))$. This allows us to use binary search in order to find matching subintervals. The complete algorithm is sketched in Algorithm 3.

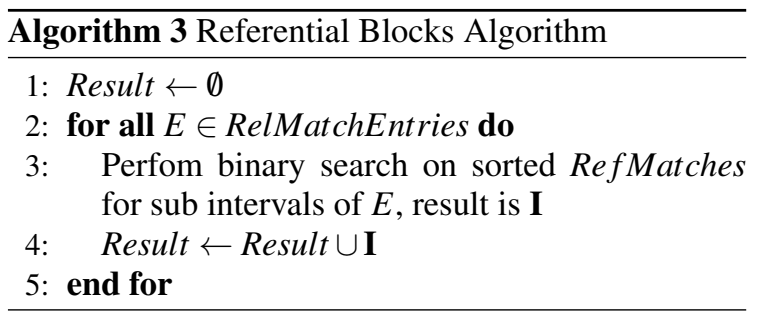

In our example, we have identified the block $\operatorname{Match}(6,7)$ as the only referential block containing the substring for $A C C$. The position of $q$ inside a referential block can be obtained by subtracting the beginning of the reference match (in our example 6) from the position of the relative match entry (also 6, which yields position 0 ).

\subsection{Finding Matches inside Raw Blocks}

The next class of potential matches are all complete matches in raw blocks. By construction of our compression algorithm, raw blocks usually

- are very short (less than $M I N$ symbols) or

- contain only non-base symbols, e.g. $N$.

Therefore, direct substring search for occurrences of $q$ is efficient here. Since the query never contains any $\mathrm{N}$ symbols, matches in raw blocks are easy to find.

In our example, we have to check all raw blocks in RawEntries for containing the query $A C C$, Neither of the two raw entries, $\operatorname{RawB}(C C C T)$ and $\operatorname{RawB}(C C)$, contains $A C C$. Thus, after the second step we still have yet only found one (out of the three) occurrences of $A C C$ in the input sequence. 


\subsection{Finding Overlapping Matches}

The most difficult task is to find matches overlapping two or more compression blocks. We partially decompress one substring for each overlapping area of compression blocks. We apply the following steps for each compression block $B$ :

1. Decompress the last $|q|-1$ characters of $B$ into string temp

2. Further decompress the following compression entries until $\mid$ temp $|=2 *| q \mid-2$

The rationale is that an exact match starting in $B$ must match $1 \leq x \leq|q|-1$ symbols in $B$ and then $|q|-x$ symbols in the next compression blocks. Therefore, it is sufficient to partially decompress only $2 *|q|-2$ symbols in totel. If the partially decompressed string temp contains $q$, then the compressed input sequence contains $q$ as well. The position can be easily computed from the match inside the string temp and the position of the first compression block.

In our example, we have four compression entries and need to decompress three overlapping strings:

- Overlap after $\operatorname{RefB}(6,7): C A \circ C C=C A C C$,

- Overlap after $\operatorname{RawB}(C C C T): C T \circ A G=C T A G$,

- Overlap after $\operatorname{RefB}(0,5): C A \circ C C=C A C C$.

The string $C A C C$ contains the query $A C C$ and therefore we have found two additional matches (one starting at the end of the first compression block and one starting at the end of the third compression block). The matching position inside the input can be computed from the matching position inside the overlap string, yielding 6 and 15 .

Combining the above results we obtain the following three positions as matches for the query $A C C$ : $\{0,6,15\}$.

\section{Evaluation}

In the following section, we evaluate our proposed compression and string search using different settings. All experiments have been run on an Acer Aspire 5950G with 16 GB RAM and eight Intel Core i7-2670QM, on Fedora 16 (64-Bit, Linux kernel 3.1). All size measures are in byte, e.g. $1 \mathrm{MB}$ means $1,000,000$ bytes.

As test data we have used $\mathrm{HG} 19 \mathrm{H}($ Kent et al., 2002) as a reference and the Korean genome(Ahn et al., 2009) as input. The Korean genome contains, besides bases and $\mathrm{N}$-symbols additional characters for indicating base probabilities. In related work it is common practice to replace all occurrences of these additional characters by $\mathrm{N}$, which yields lossy compression. However, we have chosen to encode the original sequence, in order to obtain lossless compression. Our compression rate could be further improved, if these additional symbols were replaced by $\mathrm{N}$. The compression time for a complete human genome was 29 seconds. To the best of our knowledge this is almost one order of magnitude faster than the best existing approach for referential compression. Since our algorithms can be easily parallelized, we think that the performance of our simple compression algorithm can be improved further.

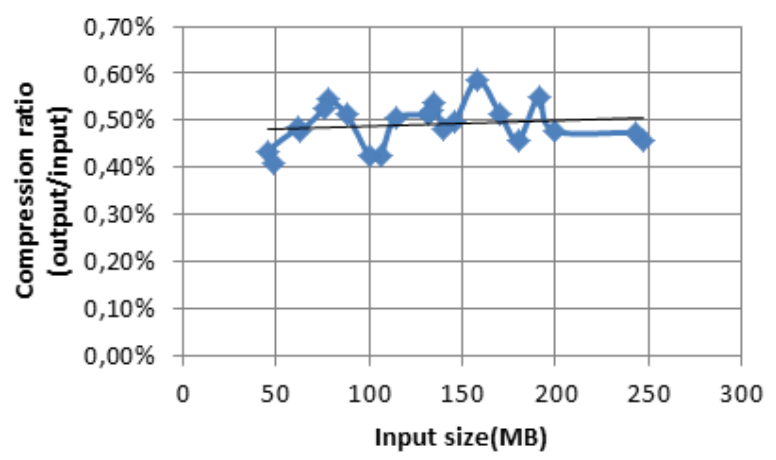

Figure 3: Compression ratio

In Figure 3, the compression ratio is shown for several data sequences (different chromosomes). The compression ratio (size of the compressed output divided by the size of the input) is roughly independent of the size of the input, and is around 0.50 percent. This means that we achieve a 20 -fold compression. The average length of referential blocks is shown in Figure 4. It can be seen that the average length of referential blocks is also roughly independent of the input chromosome and in average at 630 symbols/referential block. This coincides with the background knowledge that around 99.9 percent of two human genomes are equal.

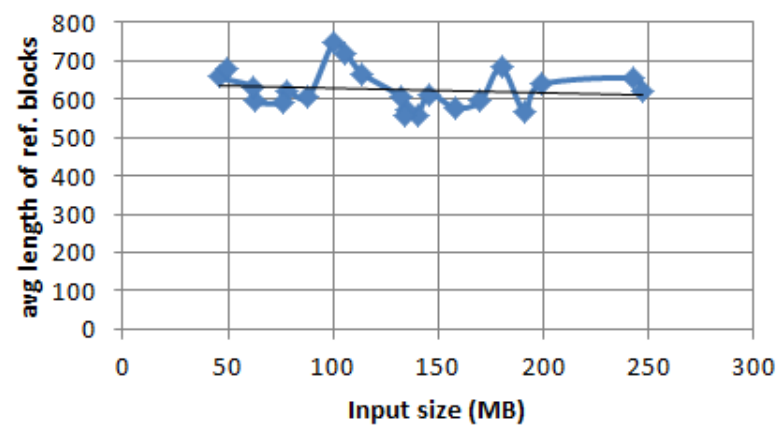

Figure 4: Average length of ref. blocks

Further analysis of the compression blocks 
showed that the average length of raw blocks is 17 symbols. 54 percent of the blocks in a compressed file are referential blocks and the remaining 46 percent are raw blocks.

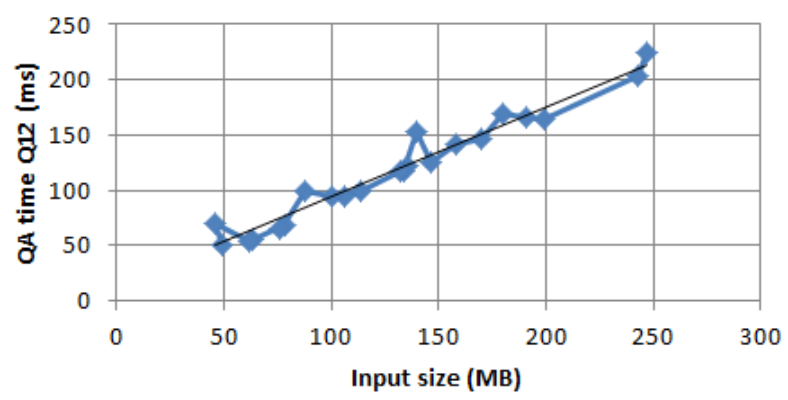

Figure 5: Answering times for $Q 12$

In order to evaluate our string search algorithm, we have measured the run time for string search for several queries. The result for a query string of length 12 is shown in Figure 5. The time needed for string search grows linear with the length of the input sequence. This is due to the (linear) traversal of all compression blocks during the search phase.

We ran experiments with different query lengths: $6,12,24,48$, and 96. Longer queries seem to slow down the string search a little bit (order of few ms comparing length 12 with length 96). Our investigation show that this is caused by the implementation of compressed suffix trees we have used: for longer queries, the library spends more time to lookup matches in the reference sequence.

Since, to the best of our knowledge, there exists no related work on string search over referentially compressed genome sequences, we have implemented the following competitors ourself for evaluation purposes:

1. Naive search: First we completely decompress the compressed input sequence into main memory and then search in-memory without an index, using bit-parallel string matching (Peltola and Tarhio, 2003).

2. Index-based search: We use an existing suffix tree for the uncompressed input sequence, in order to find string matches.

The results are shown in Figure 6. It can be seen that the naive approach is the slowest one, using almost 2 seconds to lookup the test string in the input chromosome (150 MB). Therefore, we think that decompression is no solution for string matching with respect to compressed files. The approach using suffix trees is superior to our new approach, taking 106 $\mathrm{ms}$ compared to $125 \mathrm{~ms}$. However, there are concerns:

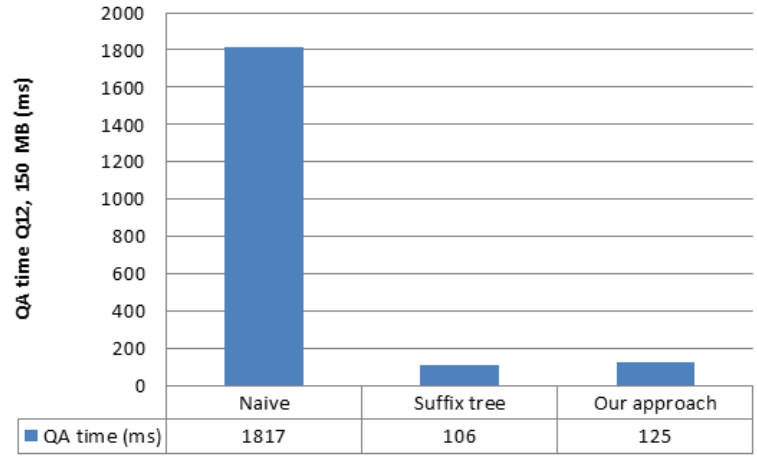

Figure 6: Answering times for $Q 12$

1. The suffix tree of the index has to be created beforehand/offline. The additional time, we measured 10-40 seconds per chromosome for our test genome, should be taken into account.

2. The suffix tree uses a lot of space. We measured roughly an increase of factor 2-4 for our test chromosomes. This means, that the index structure is even bigger than the original uncompressed file.

Since suffix trees, or alternative index structures, have to be created for each(!) input sequence, we think that the small time overhead of our proposed approach is clearly acceptable. No extra data structures have to be computed and stored offline. All we use is the compressed file and the (one) index structure of the reference sequence.

\section{Conclusions and Future Work}

One of the challenges in managing sequence data is efficient storage and retrieval over compressed data. In this paper, we addressed this problem by proposing an algorithm for string search, which works directly on referentially compressed genome sequences. Our evaluation shows that we can achieve similar run times as if we had an index structure for each compressed sequence. The ability to search biological sequences directly in a compressed structure opens new ways for managing data in research groups. For instance, a main-memory genome database, where all genomes can be hold in RAM.

One important open challenge is approximate string searching over referentially compressed sequences. We think that our search scheme can be extended in order to find approximate matches as well.

Scientific workflows have gained increased interest during the last years in biology. The integration of referential compression and string searching into these workflows is one further open challenge. 


\section{REFERENCES}

Ahn, S.-M., Kim, T.-H., Lee, S., Kim, D., Ghang, H., Kim, D.-S., Kim, B.-C., Kim, S.-Y., Kim, W.-Y., Kim, C., Park, D., Lee, Y. S., Kim, S., Reja, R., Jho, S., Kim, C. G., Cha, J.-Y., Kim, K.-H., Lee, B., Bhak, J., and Kim, S.-J. (2009). The first Korean genome sequence and analysis: Full genome sequencing for a socio-ethnic group. Genome Research, 19(9):1622-1629.

Altschul, S. F., Gish, W., Miller, W., Myers, E. W., and Lipman, D. J. (1990). Basic local alignment search tool. Journal of molecular biology, 215(3):403-410.

Antoniou, D., Theodoridis, E., and Tsakalidis, A. (2010). Compressing biological sequences using self adjusting data structures. In Information Technology and Applications in Biomedicine.

Bharti, R. K., Verma, A., and Singh, R. (2011). A biological sequence compression based on cross chromosomal similarities using variable length lut. International Journal of Biometrics and Bioinformatics, 4:217-223.

Bhola, V., Bopardikar, A. S., Narayanan, R., Lee, K., and Ahn, T. (2011). No-reference compression of genomic data stored in fastq format. In $B I B M$, pages $147-150$.

Boyer, R. S. and Moore, J. S. (1977). A fast string searching algorithm. Commun. ACM, 20(10):762-772.

Brandon, M. C., Wallace, D. C., and Baldi, P. (2009). Data structures and compression algorithms for genomic sequence data. Bioinformatics, 25(14):1731-1738.

Chen, W., Lu, Y., Lai, F., Chien, Y., and Hwu, W. (2011). Integrating human genome database into electronic health record with sequence alignment and compression mechanism. J Med Syst.

Chiang, G.-T., Clapham, P., Qi, G., Sale, K., and Coates, G. (2011). Implementing a genomic data management system using iRODS in the Wellcome Trust Sanger Institute. BMC Bioinformatics, 12(1):361+.

Daily, K., Rigor, P., Christley, S., Xie, X., and Baldi, P. (2010). Data structures and compression algorithms for high-throughput sequencing technologies. BMC bioinformatics, 11(1):514+.

Deorowicz, S. and Grabowski, S. (2011). Robust Relative Compression of Genomes with Random Access. Bioinformatics.

Duc Cao, M., Dix, T. I., Allison, L., and Mears, C. (2007). A simple statistical algorithm for biological sequence compression. In Proceedings of the 2007 Data Compression Conference, pages 43-52, Washington, DC, USA. IEEE Computer Society.

Grabowski, S. and Deorowicz, S. (2011). Engineering relative compression of genomes. CoRR, abs/1103.2351.

Hunt, E., Atkinson, M. P., and Irving, R. W. (2002). Database indexing for large dna and protein sequence collections. The VLDB Journal, 11(3):256-271.

Kahn, S. D. (2011). On the future of genomic data. Science, 331(6018):728-729.

Kaipa, K. K., Bopardikar, A. S., Abhilash, S., Venkataraman, P., Lee, K., Ahn, T., and Narayanan, R. (2010). Algorithm for dna sequence compression based on prediction of mismatch bases and repeat location. In Bioinformatics and Biomedicine Workshops (BIBMW).

Kent, W. J. (2002). BLATThe BLAST-Like Alignment Tool. Genome Research, 12(4):656-664.

Kent, W. J., Sugnet, C. W., Furey, T. S., Roskin, K. M., Pringle, T. H., Zahler, A. M., and Haussler, D. (2002). The human genome browser at UCSC. Genome Res, 12(6):996-1006.

Kuruppu, S., Beresford-Smith, B., Conway, T., and Zobel, J. (2012). Iterative dictionary construction for compression of large dna data sets. IEEE/ACM Trans. Comput. Biol. Bioinformatics, 9(1):137-149.

Kuruppu, S., Puglisi, S. J., and Zobel, J. (2010). Relative lempel-ziv compression of genomes for large-scale storage and retrieval. In Proceedings of the 17th international conference on String processing and information retrieval, SPIRE'10, pages 201-206, Berlin, Heidelberg. SpringerVerlag.

Mishra, K. N., Aaggarwal, D. A., Abdelhadi, D. E., and Srivastava, D. P. C. (2010). An efficient horizontal and vertical method for online dna sequence compression. International Journal of Computer Applications, 3(1):39-46. Published By Foundation of Computer Science.

Pande, P. and Matani, D. (2011). Compressing the human genome against a reference. Technical report, Stony Brook University.

Peltola, H. and Tarhio, J. (2003). Alternative algorithms for bit-parallel string matching. In SPIRE, pages 80-94.

Pennisi, E. (2011). Will Computers Crash Genomics? Science, 331(6018):666-668.

Pratas, D. and Pinho, A. J. (2011). Compressing the human genome using exclusively markov mod- 
els. In Rocha, M. P., Rodrguez, J. M. C., FdezRiverola, F., and Valencia, A., editors, $P A C B B$, volume 93 of Advances in Intelligent and Soft Computing, pages 213-220. Springer.

Schadt, E. E., Turner, S., and Kasarskis, A. (2010). A window into third-generation sequencing. $\mathrm{Hu}$ man molecular genetics, 19(R2):R227-R240.

Ukkonen, E. (1995). On-Line Construction of Suffix Trees. Algorithmica, 14(3):249-260.

Vey, G. (2009). Differential direct coding: a compression algorithm for nucleotide sequence data. The Journal of Biological Database and Curation, 2009.

Vlimki, N., Mkinen, V., Gerlach, W., and Dixit, K. (2009). Engineering a compressed suffix tree implementation. ACM Journal of Experimental Algorithmics, 14.

Wan, R., Anh, V. N., and Asai, K. (2011). Transformations for the compression of fastq quality scores of next generation sequencing data. Bioinformatics. 